\title{
On the normalized turbulent energy dissipation rate
}

\author{
P. Burattini, ${ }^{\text {a) }}$ P. Lavoie, and R. A. Antonia \\ Discipline of Mechanical Engineering, University of Newcastle, 2308 NSW, Australia
}

(Received 28 June 2005; accepted 9 August 2005; published online 16 September 2005)

This paper revisits values of the normalized energy dissipation rate $\left(C_{\epsilon}\right)$ in different flows (two-dimensional wakes, grid turbulence, and homogeneous shear flow). Previously published as well as new data are considered over a relatively wide range of the Taylor-microscale Reynolds number $R_{\lambda}$. $C_{\epsilon}$ exhibits wide scatter (in the range $0.5-2.5$ for $R_{\lambda}>50$ ) although, for a given flow and initial conditions, it is independent of $R_{\lambda}$ when the latter is sufficiently large. An alternative definition [B. R. Pearson, P.- $\AA$. Krogstad, and W. van de Water, "Measurements of the turbulent energy dissipation rate," Phys. Fluids 14, 1288 (2002)] of $C_{\epsilon}$ has been checked in the same flows but has failed to yield a universal value for the coefficient. (C) 2005 American Institute of Physics. [DOI: 10.1063/1.2055529]

The dependence of the turbulent energy dissipation rate $\langle\epsilon\rangle$ (here, angular brackets denote averaging with respect to time) on the Taylor-microscale Reynolds number $R_{\lambda}$ has been studied extensively. Frisch ${ }^{1}$ noted that the constancy of $C_{\epsilon}\left(\equiv\langle\epsilon\rangle L /\left\langle u^{2}\right\rangle^{3 / 2}\right.$, where $3\left\langle u^{2}\right\rangle / 2$ is the mean turbulent kinetic energy and $L$ the integral length scale) at large enough $R_{\lambda}$ is one of the two experimental laws of fully developed turbulence. Others ${ }^{2,3}$ refer to it as the zeroth law of turbulence. Kolmogorov implicitly assumed that, for large values of $R_{\lambda}$, the mean energy dissipation rate should be independent of the viscosity. Thus, for a fixed turbulent kinetic energy, $C_{\epsilon}$ should attain a constant value as the viscosity is decreased, i.e., as $R_{\lambda}$ is increased. Batchelor ${ }^{4}$ tried to verify this hypothesis in grid turbulence, albeit for a restricted range of low $R_{\lambda}$. While there is general consensus that $C_{\epsilon}$ becomes constant for moderately large $R_{\lambda}$ ( $\gtrsim 50$, e.g., Ref. 5), the actual asymptotic values of $C_{\epsilon}$ in different turbulent flows such as wakes, jets, grid turbulence, and in numerical simulations of box turbulence display a large scatter, e.g., Refs. 6-8. Even for grid turbulence, which is to a reasonable approximation homogeneous and isotropic, differences arise between different types of grids. ${ }^{6} \mathrm{~A}$ unique value of $C_{\epsilon}$ would imply that the small scales are independent of the viscosity, in the limit of large $R_{\lambda}$.

In this paper, we consider new as well as published data of $C_{\epsilon}$ for different $R_{\lambda}$ and in several flow types. This collection of data represents an update of the data in Ref. 6 (which are not repeated here) and aims at assessing the influence of initial conditions on the values of $C_{\epsilon}$, a point left open by Sreenivasan. ${ }^{6,7}$ The other purpose is to evaluate a recent suggestion for an alternate definition of the dissipation rate coefficient. Pearson et al. ${ }^{9}$ proposed that $C_{\epsilon}$ should be redefined using the integral length scale $\left(L_{p}\right)$ derived from the inverse of the wave number $\left(L_{p} \equiv 1 / k_{1 p}\right)$ at which the onedimensional (1D) longitudinal energy spectrum premultiplied by the longitudinal wave number (i.e., $k_{1} E_{11}$ ) is maximum. According to these authors, the resulting coefficient,

\footnotetext{
${ }^{a)}$ Telephone: +61 (0)2 4921 8879. Fax: +61 (0)2 4921 6946. Electronic mail: Paolo.Burattini@newcastle.edu.au
}

$C_{\epsilon}^{p}\left(\equiv\langle\epsilon\rangle L_{p} /\left\langle u^{2}\right\rangle^{3 / 2}\right)$, is independent of the flow and is close to 0.5 , when $R_{\lambda} \geq 300$. We recall that, in experiments, the integral length scale $(L)$, which features in $C_{\epsilon}$, is usually defined as

$$
L \equiv \int_{0}^{r_{0}} \rho_{u u}\left(r_{1}\right) d r_{1}
$$

where $\rho_{u u}\left(r_{1}\right) \equiv\left\langle u\left(x_{1}\right) u\left(x_{1}+r_{1}\right)\right\rangle /\left\langle u^{2}\right\rangle$ is the autocorrelation coefficient of the longitudinal velocity fluctuation, the integration being usually extended to the first zero crossing $\left(r_{0}\right)$ of $\rho_{u u}$. This limit avoids the difficulty associated with unsteadiness at low wave numbers of the velocity signal, which may prevent the autocorrelation from reaching zero at large separations, much larger than the integral length scale. In isotropic turbulence and with $r_{0}$ in Eq. (1) equal to $\infty$, this definition is equivalent to

$$
L=\frac{\pi E_{11}(0)}{2\left\langle u^{2}\right\rangle},
$$

since $E_{11}$ and $\rho_{u u}$ are a Fourier pair, i.e., $E_{11}\left(k_{1}\right)$ $=2 /\left(\pi\left\langle u^{2}\right\rangle\right) \int_{0}^{\infty} \rho_{u u}\left(r_{1}\right) \cos \left(k_{1} r_{1}\right) d r_{1}$. A different definition,

$$
L \equiv \frac{\pi}{2\left\langle u^{2}\right\rangle} \int_{0}^{k_{\max }} k^{-1} E(k) d k,
$$

is almost always applied to direct numerical simulations (DNS) data for which the three-dimensional (3D) energy spectrum $E(k)$ is known, up to a maximum value $k_{\max }$ of the wave number. For homogeneous isotropic turbulence, (2) and (3) are equivalent (e.g., Ref. 10), which can be readily shown by considering the isotropic relation

$$
E(k)=\frac{1}{2} k^{3} \frac{d}{d k}\left(\left.\frac{1}{k} \frac{d E_{11}\left(k_{1}\right)}{d k_{1}}\right|_{k_{1}=k}\right)
$$

and integrating by parts.

The measurement of the mean energy dissipation rate is fraught with uncertainties, especially in connection with probe resolution and electronic noise. Further, if the turbulence is not isotropic, the 1D estimate $\langle\epsilon\rangle_{\text {iso }}=15 \nu\left\langle(\partial u / \partial x)^{2}\right\rangle$ 


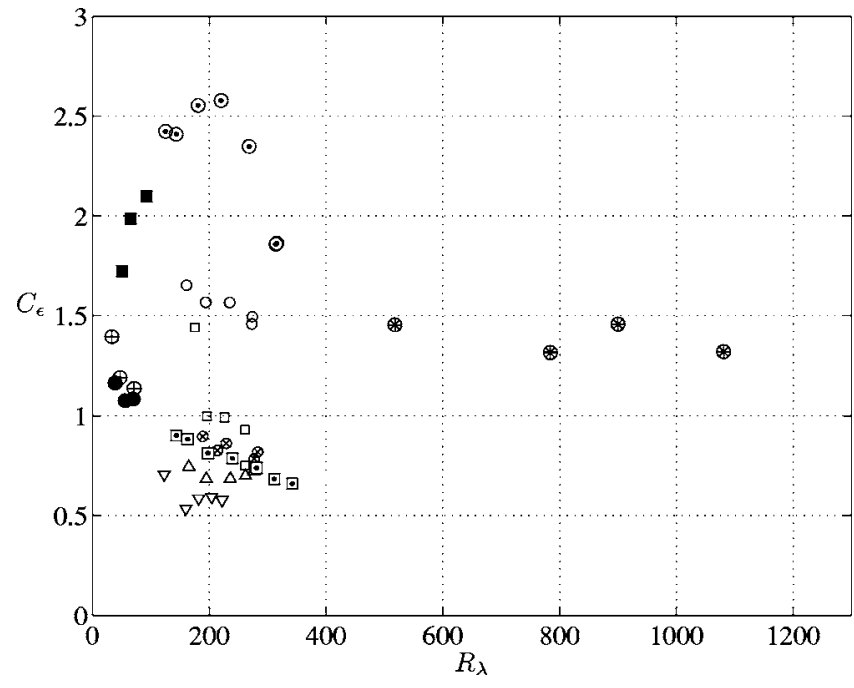

FIG. 1. Normalized turbulent energy dissipation rate $C_{\epsilon} \equiv\langle\epsilon\rangle L /\left\langle u^{2}\right\rangle^{3 / 2}$ for different flows. $\mathbf{\square}$, grid turbulence (Sq35, present data); $\oplus$, grid turbulence (Rd35, present data); $\bullet$, grid turbulence $(\mathrm{Rd} 44$, present data); $\circledast$, active-grid turbulence (from Ref. 21); $\odot$, cylinder wake (present data); $\square$, plate wake (present data); $\otimes$, round screen-cylinder wake (from Ref. 11); $\bigcirc$, round cylinder wake (from Ref. 11); $\square$, square cylinder wake (from Ref. 11); $\triangle$, plate wake (from Ref. 11); $\nabla$, screen-strip wake (from Ref. 11).

involves errors which are difficult to quantify. In this regard, grid turbulence has the advantage that $\langle\epsilon\rangle$ can be estimated indirectly, but more reliably, from the streamwise decay rate of the mean turbulent kinetic energy, $3\left\langle u^{2}\right\rangle$ (a large scale quantity), $\langle\epsilon\rangle=-(3 / 2) U d\left\langle u^{2}\right\rangle / d x$, where $U$ is the mean streamwise velocity. Another "simple" flow is homogenous shear turbulence where $\langle\epsilon\rangle$ is ideally equal to the turbulent energy production (also a large scale quantity). However, in experimental realizations of this flow, the magnitude of $\langle\epsilon\rangle$ as inferred from the energy budget can differ from its 1D isotropic estimate. The difficulties in estimating $L$ and $\langle\epsilon\rangle$ should be kept in mind when assessing the behavior of $C_{\epsilon}$.

New measurements were made in the wakes of bluff bodies with a single hot wire (diameter $d=1.27 \mu \mathrm{m}$, aspect ratio $l / d \simeq 200$, sampling frequency $f_{s}$ in the range $3-25 \mathrm{kHz}$ variable, depending on the mean velocity, low-pass filter $f_{c}$ $=f_{s} / 2$ ). A third-order polynomial was used to fit the single wire response. Two different body shapes were considered: the circular cylinder (diameter $\simeq 28.25 \mathrm{~mm}$ ) and a flat plate (width $\simeq 25 \mathrm{~mm}$ ) in the range $120 \gtrsim R_{\lambda} \gtrsim 350$. Data behind biplane, square-meshed grids in the range $20 \leqslant x / M \leqslant 80$ (M, mesh size) are also reported. Three types of grids were considered: Rd44 (round bars, solidity 44\%), Rd35 (round bars, solidity 35\%), and Sq55 (square bars, solidity 55\%). The velocity was measured with a single wire probe $(d$ $=2.54 \mu \mathrm{m}, l / d \simeq 200$ ).

Figure 1 indicates that the range of values for $C_{\epsilon}$ is quite large, typically in the range 0.5-2.5. Data from different wakes ${ }^{11}$ (circular cylinder, square cylinder, flat plate, screen strip, and a screen cylinder) and active $\mathrm{e}^{12,13}$ and passive grids have been included. If we consider each realization of either a particular wake or grid separately, then $C_{\epsilon}$ is approximately constant for $R_{\lambda} \geqslant 60$. However, even for a specific flow, differences exist depending on the details of the initial condi-

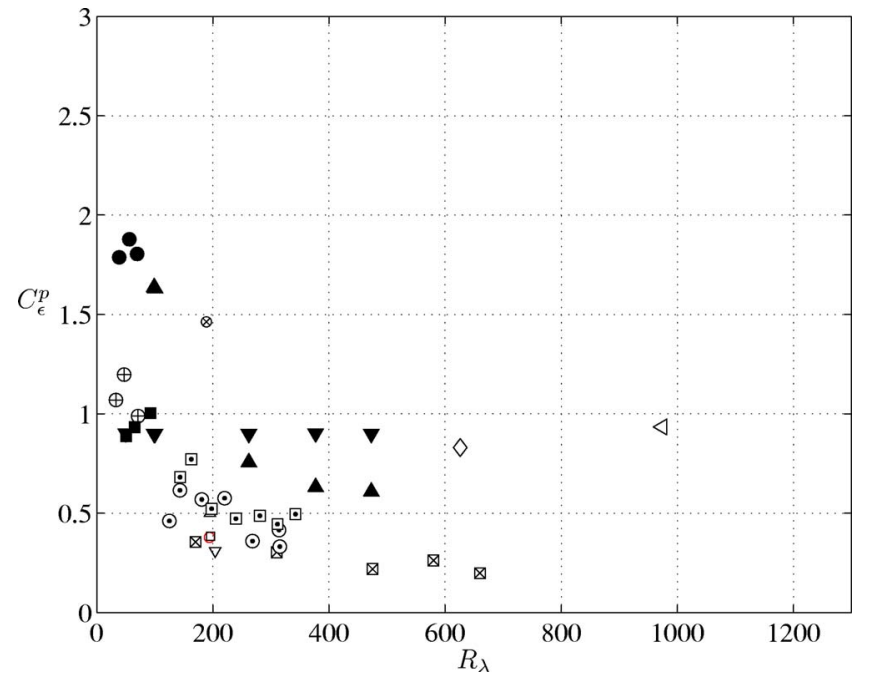

FIG. 2. Normalized turbulent energy dissipation rate $C_{\epsilon}^{p} \equiv\langle\epsilon\rangle L_{p} /\left\langle u^{2}\right\rangle^{3 / 2}$ for different flows. $\boldsymbol{\Lambda}$, active-grid turbulence [from Ref. 12 according to Pearson et al. (Ref. 9)]; $\mathbf{\nabla}$, active-grid turbulence (from Ref. 12); $\circledast$, active-grid turbulence (from Ref. 21); $\diamond$, active-grid turbulence (from Ref. 13); $\mathbf{\square}$, grid turbulence $(\mathrm{Sq} 35$, present data); $\oplus$, grid turbulence (Rd35, present data); $\bullet$, grid turbulence (Rd44, present data); $\odot$, cylinder wake (present data); $\square$, plate wake (present data); $\otimes$, round screen-cylinder wake (from Ref. 11); $\bigcirc$, round cylinder wake (from Ref. 11); $\square$, square cylinder wake (from Ref. 11); $\triangle$, plate wake (from Ref. 11); $\nabla$, screen-strip wake (from Ref. 11); $\nabla$, homogeneous shear flow (from Ref. 15); $\triangleleft$, homogeneous shear flow (from Ref. 14).

tions (e.g., the geometry of the bluff body or the shape of the grid). The present grid data are for low values of $R_{\lambda}$, so that some dependence on $R_{\lambda}$ is expected, as noted by Sreenivasan. ${ }^{6}$ More interestingly, the geometry of the grid appears to have a persistent influence in the streamwise direction up to $x / M=80$.

Figure 2 reports $C_{\epsilon}^{p}$, according to the "new" definition of the integral length scale used in Ref. 9. Some of the values of $L_{p}$ were evaluated after digitizing the original spectral data (from the references listed in the caption). Homogeneous shear flow ${ }^{14,15}$ data have also been included. The values of $C_{\epsilon}^{p}$ differ from flow to flow, with $0.2 \lesssim C_{\epsilon}^{p} \lesssim 1$ for $R_{\lambda} \gtrsim 200$. The effect of different initial conditions or the magnitude of the shear is appreciable, even though the scatter is reduced with this alternative definition. Although the 1D isotropic estimates of $\langle\epsilon\rangle$ in Refs. 14 and 15 are expected to be smaller than the values of $\langle\epsilon\rangle$ inferred from the energy budget, the uncertainty associated with $\langle\epsilon\rangle$ is unlikely to account for the difference in $C_{\epsilon}^{p}$ between the two sets of homogeneous shear flow data used in Fig. 2. A plausible explanation is that, relative to $L, L_{p}$ is more representative of smaller scales (recall that $L_{p}$ is associated with the peak of $k_{1} E_{11}$ and multiplication of $E_{11}$ with $k_{1}$ gives more weighting to larger wave numbers), and hence less sensitive to the largest (energy containing) scales, and, consequently, the initial conditions which influence primarily those scales. We have noted that the data of Mydlarski and Warhaft ${ }^{12}$ yield values of $C_{\epsilon}^{p}$ around 0.9 (using the integral length scales given in their Table 1; these are fully consistent with the definition of Pearson et al., ${ }^{9}$ as can be inferred from the discussion on pages 342 -343 of Ref. 12), and not $\simeq 0.5$, as indicated by Pearson 
et al. ${ }^{9}$ (their Fig. 1). It seems that the latter authors, when including the data of Ref. 12, assumed a constant value of $L_{p}(=0.1 \mathrm{~m})$, irrespectively of $R_{\lambda}$ (caption of their Fig. 1). Pearson et al.'s reason for preferring $L_{p}$ over $L_{u}$ is that there is a Strouhal number, associated with a "resemblance of a vortex street," ${ }^{\prime 9}$ which is related to the peak in the distribution of $k_{1} E_{11}$. This is probably relevant to the far wake of a bluff body, where coherent vortices can still be discerned. ${ }^{16,17}$ In the initial period of decay of grid turbulence, a large scale organization would not be expected. In this case, the peak of the spectrum (after multiplying by $k_{1}$ ) cannot be interpreted as a signature of periodicity in the flow. It thus seems misleading to try to reconcile the differences in the values of $C_{\epsilon}$ by resorting to a large scale periodicity that would apply to all flow types.

A further comment needs to be made with regard to the conclusion of Pearson et al. These authors used Eq. (3) for their DNS data of homogeneous isotropic turbulence. For this flow, $L$ is not necessarily equal to $L_{p}$. To show this, one needs to consider a form of $E(k)$, such as ${ }^{18}$

$$
E(k)=\frac{\left\langle u^{2}\right\rangle L C_{p}(k L)^{p}}{\left[1+\left(k L / k_{e} L\right)^{2}\right]^{p / 2+5 / 6}},
$$

where the constants $C_{p}$ and $k_{e} L$ can be expressed in terms of $p$ (the exponent of the spectrum for $k \rightarrow 0$ ) only, i.e.,

$$
C_{p}=\left[\frac{4}{\pi B\left(\frac{5}{6}, \frac{1}{2} p\right)}\right]^{1+p}\left[\frac{B\left[\frac{1}{3}, \frac{1}{2}(p+1)\right]}{3}\right]^{p},
$$

and

$$
k_{e} L=\frac{3 \pi}{4} \frac{B\left(\frac{5}{6}, \frac{1}{2} p\right)}{B\left[\frac{1}{3}, \frac{1}{2}(p+1)\right]},
$$

where $B$ is the beta function. The 1D energy spectrum, $E_{11}\left(k_{1}\right)$, can be calculated from

$$
E_{11}\left(k_{1}\right)=\int_{k_{1}}^{\infty} \frac{E(k)}{k}\left(1-\frac{k_{1}^{2}}{k^{2}}\right) d k .
$$

Using (4), the integral can be expressed in terms of the incomplete beta function, $\widetilde{B}$, for $p \geqslant 3$,

$$
\begin{aligned}
\frac{E_{11}\left(k_{1}\right)}{\left\langle u^{2}\right\rangle L}= & C_{p}\left(k_{e} L\right)^{p} \frac{1}{2} \widetilde{B}\left(\frac{5}{6}, \frac{1}{2} p ; z\right) \\
& -C_{p}\left(k_{e} L\right)^{p-2}\left(k_{1} L\right)^{2} \frac{1}{2} \widetilde{B}\left(\frac{11}{6}, \frac{p}{2}-1 ; z\right)
\end{aligned}
$$

with

$$
z=\frac{1}{1+\left[k_{1} L \frac{4}{3 \pi} \frac{B\left[\frac{1}{3}, \frac{1}{2}(p+1)\right]}{B\left(\frac{5}{6}, \frac{1}{2} p\right)}\right]^{2}} .
$$

It can be shown that the condition $\max \left[k_{1} E_{11}\left(k_{1}\right)\right]$, verified for $k_{1}=k_{1 \max }=1 / L_{p}$, implies

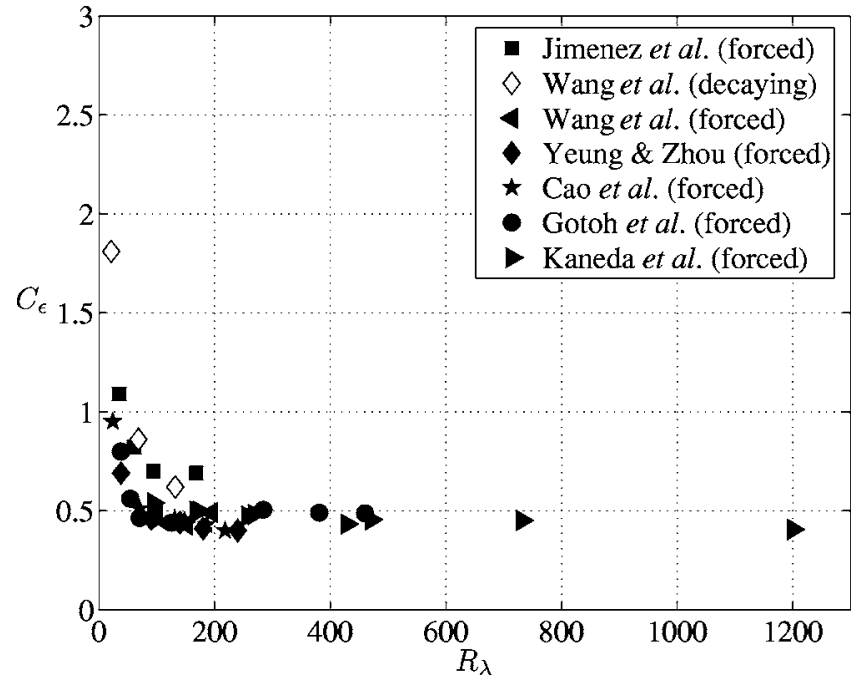

FIG. 3. Distribution of $C_{\epsilon}$ as a function of $R_{\lambda}$ for different numerical simulations of decaying and forced homogeneous isotropic turbulence (adapted from Ref. 7). $\square$, Jimenez et al. (Ref. 22); ४, $\diamond$, Wang et al. (Ref. 23); Yeung and Zhou (Ref. 24); $\star$, Cao et al. (Ref. 25);, Gotoh et al. (Ref. 20); $\gg$, Kaneda et al. (Ref. 19).

$$
\widetilde{B}\left(\frac{5}{6}, \frac{1}{2} p ; z\right)=3 \frac{\left(k_{1 \max } L\right)^{2}}{\left(k_{e} L\right)^{2}} \widetilde{B}\left(\frac{11}{6}, \frac{p}{2}-1 ; z\right),
$$

which is an implicit equation for $k_{1 \max }$. For other values of $p<3$, a numerical integration of Eq. (5) provides $E_{11}\left(k_{1}\right)$, from which the location of the maximum of $k_{1} E_{11}\left(k_{1}\right)$ can be determined. For $p=1,2,3,4, L_{p} / L$ is equal to $0.79,0.97$, $1.05,1.09$, respectively. For $p=4$, the value in the von Kármán spectrum, ${ }^{10}$ the difference between the two definitions is $10 \%$. This suggests that $L$ and $L_{p}$ are not interchangeable in homogeneous isotropic turbulence, as implied by Pearson et al., ${ }^{9}$ who assimilated $L_{p}$ to $L$ in their DNS data, even though the difference between the two definitions is relatively small in this case.

To summarize, we have reported values of the normalized energy dissipation rate in different flows and for different initial conditions. We have used two different definitions for $C_{\epsilon}$ and shown that a universal value for this coefficient is not tenable. The flow type and initial conditions (for any given flow type) seem to have a persistent influence even in the fully developed region of the flows. Part of the scatter in the published asymptotic values of $C_{\epsilon}$ is ascribable to the uncertainty in measuring the energy dissipation rate and/or to errors in estimating the integral length scale. In direct numerical simulations of box turbulence, ${ }^{7,19,20}$ where all the quantities can arguably be evaluated more reliably, $C_{\epsilon}$ is much less dispersed and seems to converge to 0.5 for $R_{\lambda}$ $\gtrsim 200$ (see Fig. 3).

The support of the Australian Research Council is gratefully acknowledged.

${ }^{1}$ U. Frisch, Turbulent Flows (Cambridge University Press, Cambridge, 1995).

${ }^{2}$ G. Eyink, Zeroth Law of Turbulence, Program on Physics of Hydrodynamic Turbulence, P. Constantin, I. Procaccia, B. Shraiman, and K. Sreenivasan, coordinators (2000): available from http:// online.itp.ucsb.edu/online/hydrot00/eyink/ 
${ }^{3}$ B. R. Pearson, T. A. Yousef, N. E. L. Haugen, A. Brandenburg, and P.- $\AA$. Krogstad, The "zeroth law" of turbulence in steady isotropic turbulence, in 15th Australasian Fluid Mechanics Conference, edited by M. Behnia, W. Lin, and G. D. McBain, paper N. AFMC00040 (The University of Sydney, 2004).

${ }^{4}$ G. K. Batchelor, The Theory of Homogeneous Turbulence (Cambridge University Press, Cambridge, 1953).

${ }^{5}$ J. L. Lumley, "Some comments on turbulence," Phys. Fluids A 4, 203 (1992).

${ }^{6}$ K. R. Sreenivasan, "On the scaling of the turbulence energy dissipation rate," Phys. Fluids 27, 1048 (1984).

${ }^{7}$ K. R. Sreenivasan, "An update on the energy dissipation rate in isotropic turbulence," Phys. Fluids 10, 528 (1998).

${ }^{8}$ R. A. Antonia and B. R. Pearson, "Effect of initial conditions on the mean energy dissipation rate and the scaling exponent," Phys. Rev. E 62, 8086 (2000).

${ }^{9}$ B. R. Pearson, P.-Å. Krogstad, and W. van de Water, "Measurements of the turbulent energy dissipation rate," Phys. Fluids 14, 1288 (2002).

${ }^{10}$ S. B. Pope, Turbulent Flows (Cambridge University Press, Cambridge, 2000).

${ }^{11}$ R. A. Antonia, T. Zhou, and G. P. Romano, "Small-scale turbulence characteristics of two-dimensional bluff body wakes," J. Fluid Mech. 459, 67 (2002).

${ }^{12}$ L. Mydlarski and Z. Warhaft, "On the onset of high-Reynolds-number grid-generated wind tunnel turbulence," J. Fluid Mech. 320, 331 (1996).

${ }^{13}$ H. S. Kang, S. Chester, and C. Meneveau, "Decaying turbulence in an active-grid-generated flow and comparisons with large-eddy simulation," J. Fluid Mech. 480, 129 (2003).

${ }^{14} \mathrm{X}$. Shen and Z. Warhaft, "The anisotropy of the small scale structure in high Reynolds number $\left(R_{\lambda} \sim 1000\right)$ turbulent shear flow," Phys. Fluids 12, 2976 (2000).

${ }^{15} \mathrm{M}$. Ferchichi and S. Tavoularis, "Reynolds number effects on the fine structure of uniformly sheared turbulence," Phys. Fluids 12, 2942 (2000).

${ }^{16}$ A. K. M. F. Hussain and M. Haykawa, "Eduction of large-scale organised structures in a plane turbulent wake," J. Fluid Mech. 180, 193 (1987).

${ }^{17}$ R. A. Antonia, L. W. B. Browne, D. K. Bisset, and L. Fulachier, "A description of the organized motion in the turbulent far-wake of a cylinder at low Reynolds number," J. Fluid Mech. 184, 423 (1987).

${ }^{18} \mathrm{H}$. Wang and W. K. George, "The integral scale in homogeneous isotropic turbulence,” J. Fluid Mech. 459, 429 (2002).

${ }^{19}$ Y. Kaneda, T. Ishihara, M. Yokokawa, K. Itakura, and A. Uno, "Energy dissipation rate and energy spectrum in high resolution direct numerical simulations of turbulence in a periodic box," Phys. Fluids 15, L21 (2003).

${ }^{20}$ T. Gotoh, D. Fukayama, and T. Nakano, "Velocity field statistics in homogeneous steady turbulence obtained using a high-resolution direct numerical simulation," Phys. Fluids 14, 1065 (2002).

${ }^{21}$ J. V. Larssen and W. J. Devenport, "The generation of high Reynolds number homogeneous turbulence," AIAA Paper 2002-2861 (2002).

${ }^{22}$ J. Jimenez, A. A. Wray, P. G. Saffman, and R. S. Rogallo, "The structure of intense vorticity in homogeneous isotropic turbulence," J. Fluid Mech. 255, 65 (1993).

${ }^{23}$ L. P. Wang, S. Chen, J. G. Brasseur, and J. C. Wyngaard, "Examination of hypotheses in the Kolmogorov refined turbulence theory through high resolution simulations. Part 1. Velocity field," J. Fluid Mech. 309, 113 (1996).

${ }^{24} \mathrm{P}$. K. Yeung and Y. Zhou, "On the universality of the Kolmogorov constant in numerical simulations of turbulence," Phys. Rev. E 56, 1746 (1997).

${ }^{25}$ N. Cao, S. Chen, and G. D. Doolen, "Statistics and structures of pressure in isotropic turbulence,” Phys. Fluids 11, 2235 (1999). 